\title{
Critical phenomena on heterogeneous small-world networks
}

\author{
Massimo Ostilli $^{1,2}$ And José F. F. Mendes $^{1}$ \\ ' 1 Departamento de Física and I3N, Universidade de Aveiro, 3810-193 Aveiro, Portugal \\ 2 Statistical Mechanics and Complexity Center (SMC), INFM-CNR SMC, Rome, Italy.
}

PACS 05.50.+q - Lattice theory and statistics (Ising, Potts, etc.)

PACS 64.60.aq-Networks

PACS $64.60 . F-$ - Equilibrium properties near critical points, critical exponents

\begin{abstract}
We consider critical phenomena on heterogeneous small-world networks having a scale-free character but also arbitrary short-loops. After deriving the self-consistent equation for the order parameter and the critical surface, we prove that the critical behavior on complex networks is in fact infinitely robust with respect to the presence of arbitrary short-loops.
\end{abstract}

massimo.ostilli@roma1.infn.it

Introduction. - The simple but fundamental CurieWeiss equation is the prototype of all mean-field mod'els. For an Ising model with a coupling $J / N$ defined over the fully connected graph of $N$ spins, the average magnetization $m$ satisfies the celebrated equation $m=$ $\tanh (\beta J m+\beta h), \beta=1 / T$ and $h$ being the inverse tem' perature and the external field. Similarly, Ising models defined on disordered graphs with a finite fixed (regular random graph) or random connectivity Poissonian distributed (classical random graph), due to the tree-like nature of the graphs, can be still exactly solved by using the BethePeierls (BP) approach [1, and the resulting average mag' netization $m$ obeys a more complicated mean field equa'tion which, however, presents the same critical behavior of the Curie-Weiss equation with the classical critical in' dices; in particular $m$ behaves as $m \sim \tau^{1 / 2}, \tau$ being the reduced temperature. In the last 10 years, a strong renewed interest towards mean-field models came from the study of cooperative phenomena defined on complex networks, i.e., random graphs having a heterogeneous distribution of links among the sites [2]. It turns out that, unlike the above random graphs, which are homogeneous, when the distribution of links is strongly heterogeneous, as happens when the degree distribution $\mathcal{P}(k)$ of the graph follows a power law $\mathcal{P}(k) \sim k^{-\gamma}$, with an exponent $\gamma<5$, the average magnetization $m$ obeys an anomalous mean field equation giving rise to the critical behavior $m \sim \tau^{1 /(\gamma-3)}$ for $\gamma>3$, or $m \sim T^{-1 /(3-\gamma)}$ for $3>\gamma>2$ [3. As in the case of the homogeneous random graphs, also for the complex networks without short-range links, the tool to achieve the mean-field equation for $m$ is basically the
BP approach that is based on the tree-like assumption of the graph which, at least for $\gamma>3$, is locally true; in fact, if $c$ is the average connectivity, the shortest loops are of length $O(\log (N) / \log (c))$, so that in the thermodynamic limit there are no loops of finite length. The analytical study of critical phenomena on complex networks has had a huge impact on the understanding of collective behaviors such as diffusion, cooperation and percolation, in biological, social or technological contexts. However, almost all these studies assume graphs locally tree-like (or at least hyper-graphs locally tree-like [4]) which in realistic networks is almost never satisfied. Social networks, the neurons of the brain, the WWW and the Internet, are just a few examples in which the average clustering coefficient $C$ [2] is finite. More precisely, whereas networks having a hierarchical structure usually share a $k$ degreedependent clustering coefficient of the form $C(k) \sim k^{-\alpha}$, with $\alpha \sim 1$, so that the most connected (and most important) nodes are not clustered, there are other networks having $C(k) \sim O(1)$ for almost any $k$. The former class includes e.g. some social networks, language networks, the WWW, and the Internet at the autonomous system level, whereas the latter class includes the Internet at the router level, the power grid, but also the brain. As discussed in [5], the reason for this difference is related to the fact that in the second class wiring is expensive (economically or biologically) and the network, rather than hierarchically, is geographically organized. It is hence of fundamental importance to understand what is the role of a loopy topology in complex networks from the point of view of collective behavior. In particular: how does change the critical surface and - above all - are the an- 
alytical results accumulated over ten years of research in complex networks robust with respect to the presence of loops? And if yes, to what extent? Notice that the crucial question concerns especially the loops of finite length but, due to the fact that the correlation length in these models remains finite, we are not allowed to neglect these shortloops neither near the critical point, so that the above questions are quite far from being trivial.

At an intermediate level between loopy-like networks (e.g. finite-dimensional lattices) and tree-like networks, stay the so called small-world networks [6] in which we have an homogeneous distribution of links among the sites, but also a finite clustering coefficient, so that tree-like based techniques as the BP cannot be used. By using a completely different approach is however possible to solve exactly also these kind of homogeneous small-world models at least in the paramagnetic phase $(\mathrm{P})$ and to get their exact critical surface and behavior [7]. If $J_{0}$ is the coupling associated to a given graph $\left(\mathcal{L}_{0}, \Gamma_{0}\right)$, which in particular may have short-range links and short loops, and $J$ is the coupling associated to a number $N c / 2$ of uniformly spread long-range links (so that they form a classical random graph), then, for any $c>0$, the mean-field equation in these models is given by

$$
m=m_{0}\left(\beta J_{0} ; c t m+\beta h\right), \quad t \stackrel{\text { def }}{=} \tanh (\beta J),
$$

where $m_{0}\left(\beta J_{0} ; \beta h\right)$ is defined as the average magnetization of the model in the absence of the long-range connections, with a short-range coupling $J_{0}$ and in the presence of a generic external field $h$ at temperature $T=1 / \beta$. Eq. (11) is a natural generalization of the Curie-Weiss meanfield equation. It is easy to check that however, for any $J_{0} \geq 0$, the critical behavior of Eq. (1) is classical, regardless of the local topology and clustering coefficient of the network (see also [8]). In this Letter we face the natural extension of Eq. (1) towards a large class of heterogeneous small-world networks that can be considered as zero or, at most, weakly degree-degree correlated. After deriving the equation for the order parameter and the critical surface (thermal or bond-percolative), to which we devote also a couple of explicit examples, we prove that the critical behavior (thermal or bond-percolative) on these networks is never affected by the presence of a local non tree-like structure, provided the connectivity associated to such loopy structures be in turns non heterogeneous.

Ising model on complex networks with loops. The family of models we shall consider is built as follows. Let $\left(\mathcal{L}_{0}, \Gamma_{0}\right)$ be any graph, $\mathcal{L}_{0}$ and $\Gamma_{0}$ being the set of vertices $i=1, \ldots, N$ and links, respectively. Let us consider the Ising model defined on the graph $\left(\mathcal{L}_{0}, \Gamma_{0}\right)$ with a fixed coupling $J_{0}$ and in the presence of an arbitrary external field $\left\{h_{i}\right\}$

$$
H_{0}=-J_{0} \sum_{(i, j) \in \Gamma_{0}} \sigma_{i} \sigma_{j}-\sum_{i} h_{i} \sigma_{i} .
$$

We will call this the pure model. Let us now consider the model obtained by removing randomly some links of the graph $\left(\mathcal{L}_{0}, \Gamma_{0}\right)$ and by adding new links as follows. Let us indicate with $c_{0 ; i, j}=0,1$ the adjacency matrix of the new graph in which some links of $\Gamma_{0}$ have been removed. Given an ensemble $\mathcal{C}$ of random graphs $\boldsymbol{c}, \boldsymbol{c} \in \mathcal{C}$, whose links are determined by the adjacency matrix elements $c_{i, j}=0,1$, we define our heterogeneous small-world model through the following Hamiltonian

$H_{\boldsymbol{c}_{0}, \boldsymbol{c}}=-J_{0} \sum_{(i, j) \in \Gamma_{0}} c_{0 ; i, j} \sigma_{i} \sigma_{j}-h \sum_{i} \sigma_{i}-J \sum_{i<j} c_{i j} \sigma_{i} \sigma_{j}$.

The averages over the graph disorder (44)-(5) will be indicated as ${ }^{-}$. The variables $c_{i, j}$ specify whether a "longrange" link (and then a coupling $J$ ) between the sites $i$ and $j$ is present $\left(c_{i, j}=1\right)$ or absent $\left(c_{i, j}=0\right)$, whereas the variables $c_{0 ; i, j}$ specify whether a "short-range" link $(i, j) \in \Gamma_{0}$ has been removed $\left(c_{0 ; i, j}=0\right)$ or not $\left(c_{0 ; i, j}=1\right)$. Both $\left\{c_{0 ; i, j}\right\}$ and $\left\{c_{i, j}\right\}$ are assumed to be independent random variables with distributions

$$
\begin{gathered}
p_{0}\left(c_{0 ; i, j}\right)=(1-p) \delta_{c_{0 ; i, j}, 1}+p \delta_{c_{0 ; i, j}, 0}, \\
p_{i j}\left(c_{i j}\right)=f\left(p_{i}, p_{j}\right) \delta_{c_{i j}, 1}+\left(1-f\left(p_{i}, p_{j}\right)\right) \delta_{c_{i j}, 0},
\end{gathered}
$$

where $p \in[0,1]$, and the $\left\{p_{i} \geq 0\right\}$ are a set of normalized hidden variables, $\sum_{i} p_{i}=1$ [9, 10, each proportional to the average degrees $\left\{\bar{k}_{i}\right\}$ of the nodes $\mathcal{L}_{0}$ in the absence of the links $\Gamma_{0}$. Usually, both $f(\cdot, \cdot)$ and the $\left\{p_{i}\right\}$ depend on one, or more, continuous parameters $\mu \in \mathcal{I}$, and on $N$. Given the mean degree of $c, c>0$ :

$$
c=\sum_{i} \frac{\bar{k}_{i}}{N}
$$

we will assume that, for a continuous subset $\mathcal{J} \subset \mathcal{I}$, asymptotically in $N$

$$
f\left(p_{i}, p_{j}\right)=c N p_{i} p_{j} .
$$

As a probability, Eq. (7) for $f\left(p_{i}, p_{j}\right)$ will be manifestly violated in $\mathcal{I} \backslash \mathcal{J}$ whenever $c N p_{i} p_{j}>1$. Note that, if $p_{i} \neq 0$ for any given $N$ (a requirement which is true for any graph in which there are no isolated nodes), for $N \rightarrow \infty$ the terms $c N p_{i} p_{j}$ tends either to 0 or to $\infty$, therefore, in $\mathcal{I} \backslash \mathcal{J}$, the number of links $(i, j)$ for which Eq. (7) is not true for $N$ large approaches

$$
\mathcal{N}_{N} \stackrel{\text { def }}{=} \sum_{i<j} \theta\left(c N p_{i} p_{j}-1\right),
$$

where $\theta(x)=0$ or 1 if $x<0$ or $x \geq 0$, respectively. If we parameterize $\mathcal{N}_{N}$ as $\mathcal{N}_{N} \sim N^{\alpha}$, and if the probability $\mathcal{P}(k)$ to have a vertex with degree $k$ scales, for $k$ large, as $\mathcal{P}(k) \sim$ $k^{-\gamma}$, it is then easy to check that we have either $\alpha=0$ for $\gamma>3$, or $\alpha=3-\gamma$, for $\gamma \leq 3$. As is intuitively clear (and as is possible to show rigorously [11), the free energy 
of (3), defined in the usual quenched way, has a leading $O(N)$ term coming from the links satisfying Eq. (7), plus the $O\left(N^{\alpha}\right)$ rest. Therefore, in the thermodynamic limit, for $\gamma>2$, this rest becomes always negligible whereas, for $N$ large but finite, the results we below present are exact up to corrections per spin which are near 0 for $\gamma>3$, or $O\left(N^{2-\gamma}\right)$ for $2<\gamma \leq 3$.

As an example one can consider the choice

$$
f\left(p_{i}, p_{j}\right)=1-e^{-c N p_{i} p_{j}}, \quad p_{i}=\frac{i^{-\mu}}{\sum_{j \in \mathcal{L}_{0}} j^{-\mu}},
$$

where $\mu \in[0,1)$. Eqs. (9) define the static model introduced in 9. Note that the "fermionic" constraint that avoids to have multiple bonds in Eq. (5) is implicit. This constraint produces some weak dis-assortative degree-degree correlations for $\mu>1 / 2$ [12. In the thermodynamic limit $N \rightarrow \infty$, for $\mu \in(0,1)$, Eqs. (9) lead to a number of long range connections per site distributed according to a power law with mean $c$ and exponent $\gamma$ given by $\gamma=1+1 / \mu$, so that $\gamma \in(2, \infty)$. For $\mu \in(0,1 / 2)$ $(\gamma>3)$ the first of Eqs. (9) takes the simpler form (7) while for $\mu \in[1 / 2,1)(2<\gamma \leq 3)$ it can be written as Eq. (7) only when $i$ and $j$ are sufficiently distant, ij $\gg N^{2-1 / \mu}$, while for lower distances, $i j \ll N^{2-1 / \mu}$, we have $p_{i j}\left(c_{i j}=1\right) \simeq 1$.

The class of our heterogeneous small-world models given by Eqs. (3)-(5) with the only conditions $c>0$ and $\gamma>2$ in $\mathcal{I} \backslash \mathcal{J}$ is very general. Note in particular that the graph $\left(\mathcal{L}_{0}, \Gamma_{0}\right)$ is completely arbitrary and can contain closed paths of any length. We stress that the resulting network, union of the graph $\left(\mathcal{L}_{0}, \Gamma_{0}\right)$ in which each link is removed with probability $p$, with the random graph $\boldsymbol{c}$, can be seen as a gran-canonical heterogeneous generalization of the original small-world graph of Watts and Strogatz [6], though we here do not have a true rewiring. Since we let the probability $p \in[0,1]$ and the mean $c \in(0, \infty)$ arbitrary, our way to build small-world networks is more general even for the homogeneous case $p_{i} \equiv 1 / N$. However, we can always restrict our class of small-world networks to the ones having a total average connectivity which does not change with $p$. To this aim we can choose $c$ such that the number of links of the graph $c$ is equal to the number of removed links of $\left(\mathcal{L}_{0}, \Gamma_{0}\right)$. Up to corrections $O(1 / \sqrt{N})$ we can accomplish this for any sample by simply taking $c=c_{0} p$, where $c_{0}$ is the average connectivity of $\left(\mathcal{L}_{0}, \Gamma_{0}\right)$.

Self consistent equation. - In the following, we will use the label ${ }_{0}$ to specify that we are referring to the pure model with Hamiltonian (2). Let $m_{0 i}\left(\beta J_{0},\left\{\beta h_{j}\right\}\right)$ be the stable magnetization of the spin $i$ of the pure model (2) at inverse temperature $\beta$. Then the order parameter $m$ of the model (3)-(7) obeys

$$
\begin{aligned}
& m=\sum_{i} m_{0 i}\left(\beta J_{0}^{(\mathrm{F})} ;\left\{N p_{j} c t m+\beta h\right\}\right) p_{i}, \\
& t \stackrel{\text { def }}{=} \tanh (\beta J), \\
& t_{0} \stackrel{\text { def }}{=} \tanh \left(\beta J_{0}^{(\mathrm{F})}\right) \stackrel{\text { def }}{=} \tanh \left(\beta J_{0}\right)(1-p) .
\end{aligned}
$$

The meaning of the order parameter $m$ is quite natural:

$$
m=\sum_{i} p_{i} \overline{\left\langle\sigma_{i}\right\rangle},
$$

$(\langle\cdot\rangle$ thermal average) while for any correlation function $C$, and for some positive $\delta$, we have

$$
C=C_{0}\left(\beta J_{0}^{(\mathrm{F})} ;\left\{N p_{j} c t m+\beta h\right\}\right)+O\left(N^{-\delta}\right),
$$

where $C_{0}\left(\beta J_{0},\left\{\beta h_{i}\right\}\right)$ is the correlation function of the pure model (2) at inverse temperature $\beta$. The proof of (10)-(12) is almost identical to the proof of Eq. (10) 7, the only difference being related to the fact that, here, the probability $p_{i j}\left(c_{i j}\right)$ depends explicitly on the link $(i, j)$. As Eq. (1), also Eqs. (10)-(12) are exact in the $\mathrm{P}$ region and provide the exact critical surface and critical exponents, while $m$ is correct up to $O\left(\bar{k} / \overline{k^{2}}\right)$ corrections out of the $\mathrm{P}$ region. A detailed proof will be reported elsewhere [11.

As an immediate consequence of Eq. (10) we get the susceptibility $\tilde{\chi}(=\partial m / \partial(\beta h))$ of the random model

$$
\tilde{\chi}=\frac{\sum_{i} p_{i} \sum_{j} \tilde{\chi}_{0 ; i, j}\left(\beta J_{0}^{(\mathrm{F})} ;\left\{N p_{l} c t m+\beta h\right\}\right)}{1-c t N \sum_{i, j} \tilde{\chi}_{0 ; i, j}\left(\beta J_{0}^{(\mathrm{F})} ;\left\{N p_{l} c t m+\beta h\right\}\right) p_{i} p_{j}}
$$

where $\tilde{\chi}_{0 ; i, j}\left(\beta J_{0} ;\left\{\beta h_{j}\right\}\right)$ stands for the two-points connected correlation function of the pure model (2) at inverse temperature $\beta: \tilde{\chi}_{0 ; i, j} \stackrel{\text { def }}{=}\left\langle\sigma_{i} \sigma_{j}\right\rangle_{0}-\left\langle\sigma_{i}\right\rangle_{0}\left\langle\sigma_{j}\right\rangle_{0}$. Note that, even if the model (2) with $h_{i} \equiv h$ is translational invariant, the model (3)-(5) is no more translational invariant (except the case $\left.p_{i} \equiv 1 / N\right)$. Note in particular that $\tilde{\chi}$ is not proportional to the sum of the connected correlation functions. In fact, from Eqs. (11) and (12) it follows

$$
\tilde{\chi}=\sum_{i, j} p_{i}\left[\overline{\left\langle\sigma_{i} \sigma_{j}\right\rangle-\left\langle\sigma_{i}\right\rangle\left\langle\sigma_{j}\right\rangle}\right] .
$$

Critical surface (thermal and percolative). From the self-consistent Eq. (10) we see that, in the thermodynamic limit, the critical surface $\beta_{c}$ satisfies

$$
c t_{c} N \sum_{i, j} \tilde{\chi}_{0 ; i, j}\left(\beta_{c} J_{0}^{(\mathrm{F})} ; 0\right) p_{i} p_{j}=1 .
$$

For any given set $\left(J_{0}, J, p,\left\{p_{i}\right\}\right)$, Eq. (15) gives the critical surface of the model in the plane $(\beta, c)$. Furthermore, from Eq. (10) it is immediate to recognize that $\beta_{c} \leq \beta_{c 0}^{(\mathrm{F})}$, with $\beta_{c}=\beta_{c 0}^{(\mathrm{F})}$ only for $J_{0}=0$, where $\beta_{c 0}^{(\mathrm{F})}$ is the critical temperature of the pure model (2) but with coupling $J_{0}^{(\mathrm{F})}$.

The theory can be projected toward the limit $\beta \rightarrow \infty$ where we get an effective percolation theory. Here the region $\mathrm{P}$ corresponds to the region in which, in the thermodynamic limit, the parameters $\left(c, c_{0}, p\right)$ are such that no giant connected component exists $(m=0)$. Note in particular that, if $c_{0 c}$ is the percolation threshold of the pure graph $\left(\mathcal{L}_{0}, \Gamma_{0}\right)$ (if $c_{0 c}$ does not exist we can set formally 
$\left.c_{0 c}=\infty\right)$ in order to remain in such a region, the connectivity $c_{0}^{(p)}=c_{0}(1-p)$ of the graph obtained from the graph $\left(\mathcal{L}_{0}, \Gamma_{0}\right)$ in which each link has been removed at random with probability $p$, must satisfy $c_{0}(1-p) \leq c_{0 c}$, otherwise a giant connected component already exists. From Eq. (15), for $c_{0}(1-p) \leq c_{0 c}$, the equation for the percolation threshold $c_{c}$ as a function of $p$ is given by

$$
c_{c} N \sum_{i, j} \tilde{\chi}_{0 ; i, j}\left(\tanh ^{-1}(1-p) ; 0\right) p_{i} p_{j}=1,
$$

where we have used the fact that $\lim _{\beta \rightarrow \infty} \tanh \left(\beta J_{0}^{(\mathrm{F})}\right)=$ $\tanh (1-p)$. Alternatively, Eq. (16) can be rewritten in terms of only graph elements as

$$
c_{c} N \sum_{i, j}\left(\delta_{i, j}+\mathcal{N}_{0 ; i, j}^{(p)}\right) p_{i} p_{j}=1, \quad c_{0}(1-p) \leq c_{0 c}
$$

where $\mathcal{N}_{0 ; i, j}^{(p)}=1$ if, in the graph $\left(\mathcal{L}_{0}, \Gamma_{0}\right)$ from which each link has been removed at random with probability $p$, between the vertex $i$ and the vertex $j$ there exists at least a path of links, and $\mathcal{N}_{0 ; i, j}^{(p)}=0$ otherwise.

Given $p$, if the condition $\quad c_{0}(1-p) \leq c_{0 c}$ is not satisfied, then a giant connected component is present and we can set $c_{c}=0$. It is interesting to see in more details the case in which we choose $c=c_{0} p$ so that, as we vary $p$, the total connectivity is fixed and equal to $c_{0}$ (as in a rewired small-world). This study is important since it leads us to understand how the presence of short loops affects diffusion processes on general networks. In particular, a strong interest regards the question: "in the presence of short loops how does the percolation threshold change?" If we set $c=c_{0} p$, from Eq. (17) we get the percolation threshold $c_{0 c}$ as a function of the rewiring parameter $p$

$c_{0 c}^{(p)} p N \sum_{i, j}\left(\delta_{i, j}+\mathcal{N}_{0 ; i, j}^{(p)}\right) p_{i} p_{j}=1, \quad c_{0}^{(p)}(1-p) \leq c_{0 c}$.

From Eq. (18) we see that $p$ may have two effects on $c_{0 c}^{(p)}$ : one tends to decrease $c_{0 c}^{(p)}$ while the other tends to increase $c_{0 c}^{(p)}$. In general $c_{0 c}^{(p)}$ decreases with $p$ for $p$ small, while grows for $p$ larger. Let us consider the case in which $p_{i} \equiv 1$; i.e., the classical small-world (no heterogeneity). In this case Eq. (18) simplifies as

$$
c_{0 c}^{(p)} p \tilde{\chi}_{0}\left(\tanh ^{-1}(1-p) ; 0\right)=1, \quad c_{0}^{(p)}(1-p) \leq c_{0 c} .
$$

For example, if $\left(\mathcal{L}_{0}, \Gamma_{0}\right)$ is the Erdös-Rényi graph (in the canonical representation), with mean connectivity $c_{0}$, by using in Eq. (19) $\tilde{\chi}_{0}\left(\beta J_{0} ; 0\right)=\left[1-c_{0} \tanh \left(\beta J_{0}\right)\right]^{-1}$ (valid in the $\mathrm{P}_{0}$ region) we get back obviously the well known percolation threshold $c_{0 c}^{(p)}=1$, independently of the value of $p$. Depending on the problem, given $c_{0}<c_{0 c}$, in general one can be more interested in reading Eq. (17) either as an equation for $p$ or for $c$. We will see later a simple example, while further analysis and applications to the percolation problem will be reported elsewhere [11].
Critical behavior. - We prove now that, if $c>0$ and $\left(\mathcal{L}_{0}, \Gamma_{0}\right)$ is not a heterogeneous graph, then the critical behavior of the model (3)-(7) does not depend either on $\left(\mathcal{L}_{0}, \Gamma_{0}\right)$ or on $\left(J_{0}, J, p, c\right)$. In [7, we have shown this result for the homogeneous small-world case $p_{i} \equiv 1 / N$ and with $p=0$. More precisely in such a case the critical behavior has been shown to be classical mean field for $t_{0} \geq 0$, while for $t_{0}<0$ first-order phase transitions are also possible (see also [13]). Here we will restrict the analysis only to the case $t_{0} \geq 0$. First of all from Eqs. (13) and (15) we observe immediately that the critical exponents for the susceptibility, above and below the critical temperature, are both equal to 1 . Let us now turn to the analysis of the order parameter near the critical point. In the following we set $h=0$ and it is understood that we consider only the positive solution of (10) $m \geq 0$. For $J_{0}=0$ we have

$$
m_{0 i}\left(\beta J_{0}^{(\mathrm{F})} ;\left\{\beta h_{j}\right\}\right)=\tanh \left(\beta h_{i}\right)
$$

so that Eq. (10) strongly simplifies in

$$
m=g(m), \quad g(m) \stackrel{\text { def }}{=} \sum_{i} \tanh \left(N p_{i} c t m\right) p_{i} .
$$

The critical behavior of the model for $J_{0}=0$, has been already studied in [14. Eq. (21) is equal to Eq. (21) of [14. We recall that, if the distribution $\left\{p_{j}\right\}$ has a power-law character, we cannot derive the correct critical behavior of the system by simply expanding the sum $g(m)$ term by term for small $m$. As shown in [14, it is necessary to keep track of all the terms of the sum $g(m)$. This is done by evaluating the sum with the corresponding integral which gives rise to a singular term proportional to $(m)^{\gamma-2}$ plus regular terms proportional to $(m),(m)^{3}$ and so on, where $\gamma=1+1 / \mu$, if $\mu$ is the power law exponent associated to $\left\{p_{j}\right\}$. As a consequence, when we solve Eq. (21) to leading order in $m$, if $T$ and $\tau$ indicate the temperature and the reduced temperature, respectively, we get the well known anomalous mean-field behavior: $m \sim O\left(\tau^{1 / 2}\right)$ (i.e. classical mean-field) for $\gamma>5, m \sim O\left(\tau^{1 /(\gamma-3)}\right)$ for $3<\gamma<5$, and $m \sim O\left(T^{-(\gamma-2) /(3-\gamma)}\right)$ for $2<\gamma<3$. Note that the critical behavior of the order parameter $m=\sum_{i} \overline{\left\langle\sigma_{i}\right\rangle} p_{i}$ is different from the unweighted one defined as $\bar{m}=\sum_{i} \overline{\left\langle\sigma_{i}\right\rangle} / N$ when $2<\gamma<3$. In such a case, from Eq. (12) one can use $\bar{m} \sim t m$ from which it follows that $\bar{m} \sim O\left(T^{-1 /(3-\gamma)}\right)$ for $2<\gamma<3$.

Let now be $J_{0}>0$. It is clear that if the graph $\left(\mathcal{L}_{0}, \Gamma_{0}\right)$ is in turn a pure scale free graph with exponent $\gamma^{\prime}$, then the joined network will have an anomalous critical behavior characterized by the minimum between $\gamma$ and $\gamma^{\prime}$. Less obvious is to understand what happens if $\left(\mathcal{L}_{0}, \Gamma_{0}\right)$ has a finite dimensional structure or some special topology with short loops. In particular we can pose the question: "does the critical behavior change by adding, via short loops, many paths between far spins, or may the critical exponent for $m$ depend on $J_{0}$ ?" Let us consider the self-consistent equation (10) in general. The exact 
expression of $m_{0 i}\left(\beta J_{0}^{(\mathrm{F})} ;\left\{\beta h_{j}\right\}\right)$ for a generic non homogeneous external field $\left\{h_{j}\right\}$ represents a formidable task. Note that, as above mentioned, to analyze the critical behavior we cannot expand for small fields $\left\{\beta h_{j}\right\}$. However, since we have proved that, for $J_{0}^{(\mathrm{F})}>0, \beta_{c 0}^{(\mathrm{F})}>\beta_{c}$, in the region $\beta_{c 0}^{(\mathrm{F})}>\beta>\beta_{c}$ we can perform an high temperature expansion of $m_{0 i}\left(\beta J_{0}^{(\mathrm{F})} ;\left\{\beta h_{j}\right\}\right)$ in the parameter $t_{0}=\tanh \left(\beta J_{0}^{(\mathrm{F})}\right)$. It is then easy to see that

$$
\begin{aligned}
& m_{0 i}\left(\beta J_{0}^{(\mathrm{F})} ;\left\{\beta h_{j}\right\}\right)=\tanh \left(\beta h_{i}\right) \\
& +t_{0}\left[1-\tanh ^{2}\left(\beta h_{i}\right)\right] \sum_{j \in \mathcal{N}_{0}(i)} \tanh \left(\beta h_{j}\right)+O\left(t_{0}^{2}\right),
\end{aligned}
$$

where $\mathcal{N}_{0}(i)$ is the set of the first neighbors of the vertex $i$ in the graph $\left(\mathcal{L}_{0}, \Gamma_{0}\right)$. Plugging Eq. (22) into Eq. (10)

$$
m=g(m)+\Delta_{1}(m)+\Delta_{2}(m),
$$

where we have introduced

$$
\Delta_{1}(m) \stackrel{\text { def }}{=} t_{0} \sum_{i} p_{i} \sum_{j \in \mathcal{N}_{0}(i)} \tanh \left(N p_{j} c t m\right),
$$

and $\Delta_{2}(m)$ is the rest coming from Eq. (22) which, near the critical point, $\left|\Delta_{2}(m)\right| \ll \Delta_{1}(m)$. Let us compare the bigger contribution $\Delta_{1}(m)$ with the "pure $J_{0}=0$ term" $g(m)$ and let us focus on the simpler cases in which the graph $\left(\mathcal{L}_{0}, \Gamma_{0}\right)$ has a fixed connectivity $\left|\mathcal{N}_{0}(i)\right| \equiv c_{0}$. Let us suppose first that $c_{0}=1$ (i.e., $\left(\mathcal{L}_{0}, \Gamma_{0}\right)$ is an ensemble of dimers). In general, given any normalized distribution $p_{i} \geq 0$, and any function $f(x) \geq 0$ increasing with $x$, the following strict (for $p_{i} \neq 1 / N$ ) inequality holds

$$
\sum_{i} p_{i} f\left(p_{j_{0}(i)}\right)<\sum_{i} p_{i} f\left(p_{i}\right),
$$

where $j_{0}(i)$ stands for the first single neighbor of $i$ in $\left(\mathcal{L}_{0}, \Gamma_{0}\right)$. Note that $i \rightarrow j_{0}(i)$ is a bijection on $\mathcal{L}_{0}$ and that $j_{0}(i) \neq i$. We can however formally enlarge the definition of $j_{0}(i)$ to include also the case $j_{0}(i)=i$. The inequality (25) tells us that when we choose $j_{0}(i)=i$ we get an optimal overlap between the distribution $\left\{p_{i}\right\}$ and the function $f(\cdot)$. For the general case $\left|\mathcal{N}_{0}(i)\right| \equiv c_{0} \geq 1$, for any $i$, we can enumerate the $c_{0}$ neighbors of $i$ in the same way as $j_{0}^{(1)}(i), \ldots, j_{0}^{\left(c_{0}\right)}(i)$, so that for each upper index $l$ (that represents an oriented direction) $j_{0}^{(l)}(i)$ is a bijection on $\mathcal{L}_{0}$ on which we can apply (25) to get finally

$$
\sum_{i} p_{i} \sum_{j \in \mathcal{N}_{0}(i)} f\left(p_{j}\right)=\sum_{l=1}^{c_{0}} \sum_{i} p_{i} f\left(p_{j_{0}^{(l)}(i)}\right)<c_{0} \sum_{i} p_{i} f\left(p_{i}\right) .
$$

By applying this equation to our case with $f(x)=\tanh (x)$ and for $t_{0}>0$ we see that for any $m>0$

$$
0<\left|\Delta_{2}(m)\right|<\Delta_{1}(m)<t_{0} c_{0} g(m) .
$$

In conclusion from Eqs. (10) and (26) we get

$g(m)<m<\left(1+t_{0} c_{0}\right) g(m)+O\left(t_{0}^{2}\right) g(m)+o(g(m))$, where $o(x)$ is such that $\lim _{x \rightarrow 0} o(x) / x=0$. In general, for $t_{0}$ finite, it is possible to prove that [1].

$$
g(m)<m<\tilde{\chi}_{0}\left(\beta J_{0}^{(\mathrm{F})} ; 0\right) g(m)+o(g(m)),
$$

where $\tilde{\chi}_{0}\left(\beta J_{0}^{(\mathrm{F})} ; 0\right)$ is the susceptibility of the pure model (2) with coupling $J_{0}^{(\mathrm{F})}$ and $h_{i} \equiv 0$. Since near the critical point, in the region $\beta_{c 0}^{(F)}>\beta>\beta_{c}$, it is $\tilde{\chi}_{0}\left(\beta J_{0}^{(\mathrm{F})} ; 0\right)<\infty$, we see that Eq. (27) implies that the critical behavior of Eq. (10) remains always that corresponding to the term $g(m)$, i.e. as if it were $J_{0}=0$. Hence, g.e., if $\left(\mathcal{L}_{0}, \Gamma_{0}\right)$ is a $d_{0}$-dimensional lattice and $\left\{p_{i}\right\}$ generates a scale-free graph with degree exponent $3<\gamma<5$, Eq. (27) tells us that near the critical temperature for the model (3) $m \sim \tau^{1 /(\gamma-3)}$, regardless the values of $J_{0}, J, p, c$ and $d_{0}$. We can also consider the case in which $\left(\mathcal{L}_{0}, \Gamma_{0}\right)$ is a Poissonian graph with mean connectivity $c_{0}$. To this aim we can start from the fully connected graph and remove from it randomly each of its $N(N-1) / 2$ links with probability $p=1-c_{0} / N$; the resulting graph will be our Poissonian graph with mean connectivity $c_{0}$. Since we have already proved that when $\left(\mathcal{L}_{0}, \Gamma_{0}\right)$ is the fully connected graph with a couplings $O(1 / N)$ the critical behavior remains equal to that of the model with $J_{0}=0$ (the effective coupling in this case being $\left.\beta J_{0}^{(\mathrm{F})}=\tanh \left(\beta J_{0}\right) c_{0} / N\right)$, we conclude that, also when $\left(\mathcal{L}_{0}, \Gamma_{0}\right)$ is a Poissonian graph, the critical behavior on the total graph remains the same as if it were $J_{0}=0$.

Examples. - Concerning the critical surface, particularly simple are the cases in which $\left(\mathcal{L}_{0}, \Gamma_{0}\right)$ is a set of disconnected finite clusters (points, dimers, triangles, .... or mixtures of them). Note that for such $\left(\mathcal{L}_{0}, \Gamma_{0}\right)$ there is no percolation threshold (or formally $c_{0 c}=\infty$ ).

Let $\left(\mathcal{L}_{0}, \Gamma_{0}\right)$ be a set of $N / 2$ disconnected dimers. This case represents the simplest example with $J_{0} \neq 0$ in which $m_{0 i}\left(\beta J_{0}^{(\mathrm{F})} ;\left\{\beta h_{j}\right\}\right)$ can be exactly calculated. We have

$$
m_{0 i}\left(\beta J_{0}^{(\mathrm{F})} ;\left\{\beta h_{j}\right\}\right)=\frac{\tanh \left(\beta h_{i}\right)+t_{0} \tanh \left(\beta h_{j_{0}(i)}\right)}{1+t_{0} \tanh \left(\beta h_{i}\right) \tanh \left(\beta h_{j_{0}(i)}\right)},
$$

where $t_{0}=\tanh \left(\beta J_{0}^{(\mathrm{F})}\right)$ and $j_{0}(i)$ stands for the first neighbor of $i$. By derivation we get the correlation function $\tilde{\chi}_{0 ; i, j}$ which, in the $\mathrm{P}$ region, takes the form

$$
\tilde{\chi}_{0 ; i, j}\left(\beta J_{0}^{(\mathrm{F})} ; 0\right)=\delta_{i, j}+t_{0} \delta_{i, j_{0}(i)} .
$$

Therefore for the critical surface we have

$$
c t_{c} N\left[\sum_{i} p_{i}^{2}+t_{0} \sum_{i} p_{i} p_{j_{0}(i)}\right]=1 .
$$

With respect to the critical surface of the model with $J_{0}=0$ we see in Eq. (30) the presence of a new term proportional to $t_{0}$. How much this term affects $t_{c}$ depends on how the dimers are placed, i.e., on how we choose the first neighbors $\left\{j_{0}(i)\right\}$. Since by definition the 
dimers are not connected, in general for $j_{0}(i)$ we can take $j_{0}(i)=i+k, \bmod N$ where $k$ is a constant integer in the range $[1, N]$. The exact evaluation of $t_{c}$ for $N$ large remains simple only if $k$ does not depend on $N$ or $k=O(N)$. Under the choice (9) for the former case we have

$$
c t_{c}\left(1+t_{c 0}\right)(1-\mu)^{2}\left(1-N^{2 \mu-1}\right)(1-2 \mu)^{-1}=1,
$$

whereas for the latter the term proportional to $t_{0}$ in the thermodynamic limit becomes negligible. Of course, we re-find that for $\mu>1 / 2($ i.e. $\gamma<3) t_{c} \rightarrow 0^{+}$.

Let $\left(\mathcal{L}_{0}, \Gamma_{0}\right)$ be now a set of $N / 3$ disconnected triangles. For simplicity here we assume $\mu=0$, i.e., the graph disorder is just the classical one (Erdös-Rényi graph) and we calculate the percolation threshold for the problem with fixed total connectivity that we have under the choice $c=c_{0} p$, with $c_{0}=2$. For the susceptibility of the pure model with generic coupling $\beta J_{0}$ we get

$$
\tilde{\chi}_{0}\left(\beta J_{0} ; 0\right)=\frac{3 e^{3 \beta J_{0}}+e^{-\beta J_{0}}}{e^{3 \beta J_{0}}+3 e^{-\beta J_{0}}} .
$$

From Eq. (19) and (32), by using the replacement $\beta J_{0} \rightarrow$ $\tanh ^{-1}(1-p)$, we get the equation for the percolation threshold $p_{c}$ (which is solved for $p_{c}=0.183406$ )

$$
2 p \frac{3 e^{3 \tanh ^{-1}(1-p)}+e^{-\tanh ^{-1}(1-p)}}{e^{3 \tanh ^{-1}(1-p)}+3 e^{-\tanh ^{-1}(1-p)}}=1 .
$$

In the above examples we had $d_{0}=0$. For a chain of $N$ spins we can evaluate $T_{c}$ from Eq. (15) by using $\tilde{\chi}_{0 ; i, j}=$ $t_{0}^{|i-j|}$. In Fig. (1) we plot simulations for the susceptibility $\chi$ as a function of $T$ for several system sizes and compare the location of the maximums with the theoretical $T_{c}$.

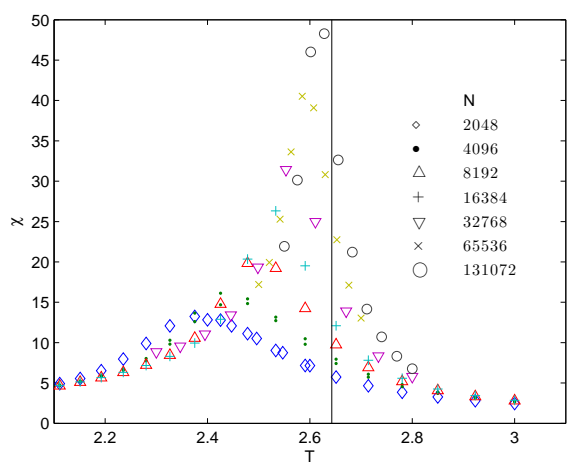

Fig. 1: Plots of $\chi$ as a function of $T$ for a chain of $\mathrm{N}$ spins in networks generated via Eqs. (9) with $c=1, J=J_{0}=1$, $\mu=1 / 3$ (corresponding to $\gamma=4$ ), and $p=0$. The vertical line comes from the solution of Eq. (15) with $N=131072$.

Conclusions. - The results presented in this Letter are easily generalized to the case in which $J_{0}$ and $J$ are random couplings and in particular we find that also for the spin-glass critical behavior a similar robustness theorem applies. These, as well as other considerations concerning the existence of strong finite size effects (see Eq. (12)) will be reported elsewhere [11. Our analysis sheds also some light to the issues related to complex networks embedded in a metric space. Although we here do not give the longrange couplings $J$ a metric structure, the graph $\left(\mathcal{L}_{0}, \Gamma_{0}\right)$ is completely general so that it can be also a metric graph equipped with an arbitrary distance $\|i-j\|_{0}$, which is the natural distance through which the correlation functions via Eq. (12) are expressed. An interesting generalization of the Ginzburg criterion for the relevance of critical fluctuations has been recently derived for Ising models built on annealed networks embedded in a metric space [15]. We recall however that the Ginsburg criterion alone, when fulfilled, does not provide the critical exponent of the order parameter $m$, especially if a heterogeneous distribution of links, and then a continuous dependence on the parameters of this distribution, is considered. At the heart of our robustness theorem (27) lies the general inequality (25) whose lhs and rhs corresponds to the short-range and to the long-range couplings of the model, respectively. We see then that the existence of long-range couplings in a model makes its order parameter already optimized with respect to the addition of any possible regular, or weakly random (i.e. Poissonian like), structure; only the addition of further long-range links sufficiently heterogeneous may change the critical behavior of the system. This work was supported by SOCIALNETS. We thank A. L. Ferreira for many useful discussion.

\section{REFERENCES}

[1] M. Mezard and G. Parisi, Eur. Phys. J. B 20, 217 (2001).

[2] R. Albert, A.L. Barabasi, Rev. Mod. Phys. 7447 (2002); S.N. Dorogovtsev, J.F.F. Mendes, Evolution of Networks (University Press: Oxford, 2003); M. E. J. Newman, SIAM Review 45, 167 (2003).

[3] S.N. Dorogovtsev, A.V. Goltsev, J.F.F. Mendes, Rev. Mod. Phys. 80, 1275 (2008).

[4] M. E. J. Newman, Phys. Rev. Lett. 103, 058701 (2009); J. P. Gleeson, Phys. Rev. E 80, 036107 (2009).

[5] E. Ravasz, A.-L. Barabasi Phys. Rev. E 67, 026112 (2003).

[6] D. J. Watts, S. H. Strogatz, Nature, 393, 440 (1998).

[7] M. Ostilli and J. F. F. Mendes, Phys. Rev. E 78, 031102 (2008).

[8] M. B. Hastings, Phys. Rev. Lett. 91, 98701 (2003).

[9] K.-I. Goh, B. Kahng and D. Kim, Phys. Rev. Lett. 87, 278701 (2001).

[10] G. Caldarelli et al., Phys. Rev. Lett. 89, 258702 (2002); M. Boguñá and R. Pastor-Satorras, Phys. Rev. E 68, 036112 (2003); J. Park and M. E. J. Newman, Phys. Rev. E 70, 066146 (2004).

[11] M. Ostilli and J. F. F. Mendes, to appear elsewhere.

[12] M. Catanzaro and R. Pastor-Satorras, Eur. Phys. J. B. 44, 241 (2005).

[13] A. L. Ferreira, M. Ostilli, J. F. F. Mendes, Phys. Rev. E 82, 011141 (2010).

[14] D.-H. Kim et al., Phys. Rev. E 71, 056115 (2005).

[15] S. Bradde et al., Phys. Rev. Lett. 104, 218701 (2010). 